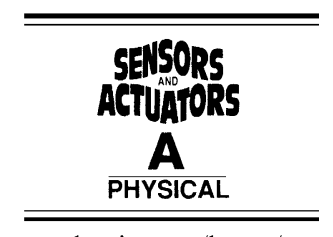

www.elsevier.com/locate/sna

\title{
Active vibration isolation of a large stroke scanning probe microscope by using discrete sliding mode control
}

\author{
Jia-Yush Yen ${ }^{\mathrm{a}, *}$, Kuo-Jung Lan ${ }^{\mathrm{a}}$, John A. Kramar ${ }^{\mathrm{b}}$ \\ ${ }^{a}$ Department of Mechanical Engineering, National Taiwan University, Taipei 10617, Taiwan \\ ${ }^{\mathrm{b}}$ National Institute of Standards and Technology, Gaithersburg, MD 20899, USA
}

Received 3 April 2004; received in revised form 15 February 2005; accepted 22 February 2005

Available online 5 April 2005

\begin{abstract}
Active vibration isolation is gaining increased attention in the ultra high precision applications to effectively treat the unavoidable ground vibration. The use of active vibration isolation is now being explored for the Molecular Measuring Machine $\left(\mathrm{M}^{3}\right)$ at the National Institute of Standards and Technology to improve its imaging resolution. The $\mathrm{M}^{3}$ system uses a Mallock suspension to establish a non-rotation constraint to the stage motion. The vibration isolation system has to work within the Mallock geometry. The system is also a six-input-six-output system; therefore, a clever design is needed to maximize the controller performance while limiting it to within the stability range. The control algorithm adopted uses discrete sliding mode control, taking advantage of its easy computer implementation and its robust high performance properties. The experimental results show that the controller is effective over the operating frequency band and is superior to the conventional lead-lag type controller.
\end{abstract}

(C) 2005 Elsevier B.V. All rights reserved.

Keywords: Discrete sliding mode control; Vibration isolation; Scanning probe microscope

\section{Introduction}

Recent advancements in nanotechnology have highlighted the need for high speed and large scanning areas from scanning probe microscopes (SPM). As the technology progresses, it has become clear that further advances in the vibration isolation of the SPM system may also be needed to improve the measurement capabilities.

Scanning probe microscopy uses a sharp, mechanical probe to raster scan the sample surface [1]. A very-high resolution piezoelectric actuator keeps a constant interaction force or constant tunneling current between the probe and the sample under servo control so that the probe can follow the sample profile variation. The size of the probe tip and the resolution of the lateral movement mechanism determine the lateral resolution capability of the system. The vertical resolution, on the other hand, is determined by the ability

\footnotetext{
* Corresponding author. Tel.: +88623366 2720; fax: +88622364 4871. E-mail address: jyen@ntu.edu.tw (J.-Y. Yen).
}

of probe to track the surface contour. Both are also subject to noise in positioning. Many research efforts have been devoted to improve the sharpness of the probe tip so that the tip can access smaller surface profile variations [2-4]. There has also been some discussion on optimizing the probe servo to maintain good tracking performance for high scanning rates [5-10]. But as the drive for improved performance continues, it has been realized that in many cases the vibrations of the measurement environment are still significantly limiting the measurement resolution. The traditional approach to vibration isolation depends heavily on a quiet foundation and very stiff structure. These approaches gradually become inadequate as the resolution improves. Notice that it is usually very difficult to improve the foundation in these cases; therefore, active vibration isolation becomes an attractive alternative.

An active suspension has been applied to the Molecular Measuring Machine $\left(\mathrm{M}^{3}\right)$ at the National Institute of Standards and Technology (NIST) [11]. The $\mathrm{M}^{3}$ system was built to measure with atomic resolution over a $50 \mathrm{~mm} \times 50 \mathrm{~mm}$ 
area [12]. This scanning area is more than 2500 times larger than most commercial SPMs. In order to achieve this large scan range, a price must be paid in terms of stiffness; therefore, vibration isolation becomes all the more essential. The suspension system in $\mathrm{M}^{3}$ is based on a Mallock suspension design, which is modified to allow active vibration isolation. Significantly, the geometry of the Mallock suspension can induce highly non-linear dynamic behavior. Therefore, a robust controller is necessary in this case. To allow for precise computer implementation, the discrete sliding mode control (DSMC) algorithm has been adopted for the $\mathrm{M}^{3}$ system. The DSMC has been the subject of many research efforts, and has proven to be a highly robust controller with very good performance [13-15]. In addition, DSMC can inherently avoid the chattering problem inherent in continuous-time sliding mode control. This research first addresses the Mallock suspension Multiple-Input-Multiple-Output (MIMO) system modeling by inserting decoupling matrices. The diagonalized model then serves as the basis for the synthesis of a MIMO controller. The implementation results show $5-10 \mathrm{~dB}$ vibration attenuation within the operation bandwidth. The comparison with conventional lead-lag compensator also demonstrates significant improvements.

This paper is divided into six sections. Section 2 describes the Mallock suspension vibration isolation system of the $\mathrm{M}^{3}$ system. Section 3 describes the diagonalized matrix. Section 4 carries the system modeling and system identification. Section 5 describes the controller design and stability analysis. Section 6 presents the implementation and the experimental results. Section 7 presents the conclusions of the paper.

\section{Active vibration isolation system}

The Mallock suspension designed by Mr. A. Mallock is the base of the $\mathrm{M}^{3}$ system suspension [16]. The Mallock suspension used for the $\mathrm{M}^{3}$ system contains an outer shell, an inner shell, six rods, and one spring, as in Fig. $1[17,18]$.

Rods $1-5$ in the figure are rigid rods so that they constrain five of the motional degrees of freedom. The spring on the top suspends the weight of the inner shell. Fig. 2 shows a photograph of the inner and outer shells.

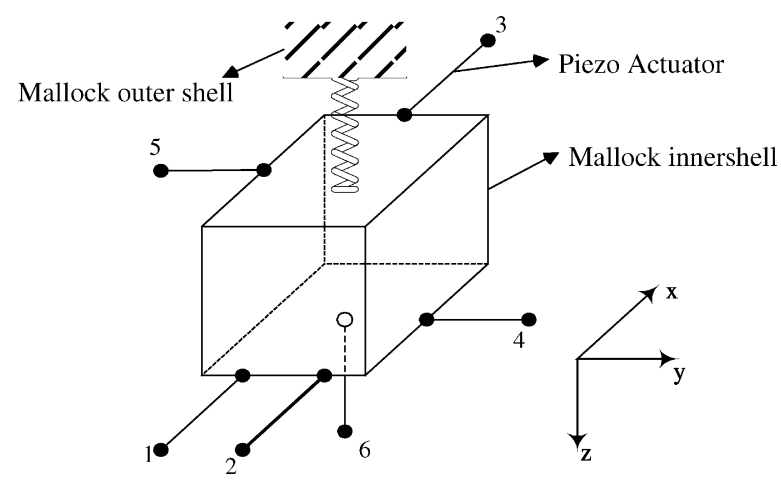

Fig. 1. A diagram for the Mallock suspension system of $\mathrm{M}^{3}$.

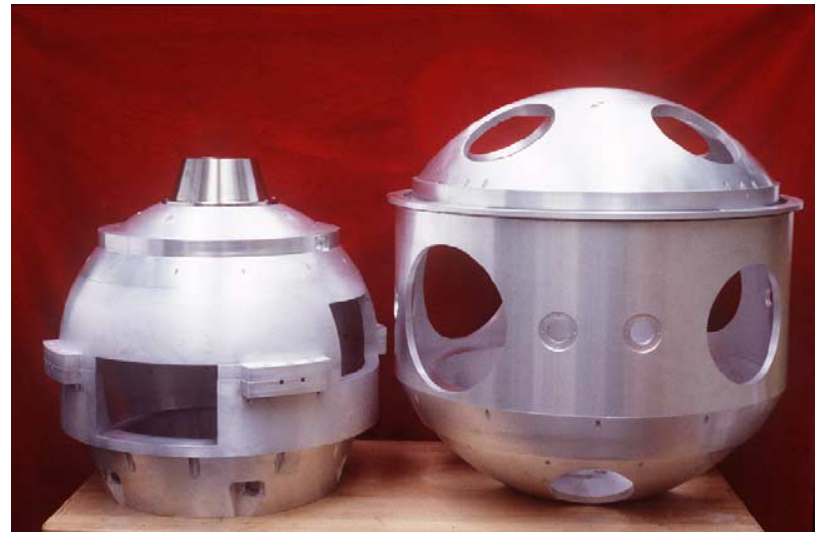

Fig. 2. The Mallock inner and outer shell photograph. In operation, the inner shell is suspended in the outer shell by means of six connecting rods.

The modified system replaces the rigid rods with piezoelectric actuators, and adds an additional actuator (number 6) to work in parallel with the spring, the spring still taking most of the static load. The first five piezoelectric actuators then control the $x$ and $y$ translation, and all three rotational motions. The sixth actuator controls the motion in the $z$ direction. The inner-shell ends of the rods are mounted with six accelerometers to detect the transmitted vibration. The accelerometers sense the vibration signal and feed the signal back to the computer for active control. One advantage of this design is that the static preload on the piezoelectric actuator is adjustable; this is accomplished by setting the tension on the springs that are placed in parallel with the piezoelectric actuators.

\section{Diagonalized matrix}

To simplify the implementation of the controller, we diagonalize the system. This procedure is used to reduce the coupling terms in the transfer function matrix. A six-by-six Multiple-Input-Multiple-Output system can be reduced to six Single-Input-Single-Output (SISO) systems. As a heuristic example, consider a two-input-two-output system. Given a transfer function, $G_{i j}(s)$, and the Laplace transform of the inputs, $U_{i}(s)$, the outputs are the product,

$$
\left[\begin{array}{l}
Y_{1}(s) \\
Y_{2}(s)
\end{array}\right]=\left[\begin{array}{ll}
G_{11}(s) & G_{12}(s) \\
G_{21}(s) & G_{22}(s)
\end{array}\right]\left[\begin{array}{l}
U_{1}(s) \\
U_{2}(s)
\end{array}\right]
$$

If one transforms the system by pre- and post-multiplying by a diagonalization matrix, the system becomes

$$
\begin{aligned}
{\left[\begin{array}{l}
\bar{Y}_{1}(s) \\
\bar{Y}_{2}(s)
\end{array}\right]=} & {\left[\begin{array}{rr}
a & b \\
c & -d
\end{array}\right]\left[\begin{array}{ll}
G_{11}(s) & G_{12}(s) \\
G_{21}(s) & G_{22}(s)
\end{array}\right]\left[\begin{array}{rr}
e & f \\
g & -h
\end{array}\right] } \\
& \times\left[\begin{array}{l}
\bar{U}_{1}(s) \\
\bar{U}_{2}(s)
\end{array}\right]
\end{aligned}
$$


$\left[\begin{array}{c}\bar{Y}_{1}(s) \\ \bar{Y}_{2}(s)\end{array}\right]=\left[\begin{array}{ll}\bar{G}_{11}(s) & \bar{G}_{12}(s) \\ \bar{G}_{21}(s) & \bar{G}_{22}(s)\end{array}\right]\left[\begin{array}{c}\bar{U}_{1}(s) \\ \bar{U}_{2}(s)\end{array}\right]$

where $a, b, c, d, e, f, g$, and $h$ are positive constants to be determined such that the determinant of diagonalized matrix is not zero. We determine these constants by maximizing the diagonal terms $\left(\bar{G}_{11}, \bar{G}_{22}\right)$ and minimizing the off diagonal terms $\left(\bar{G}_{12}, \bar{G}_{21}\right)$. Because $G_{i j}(j w)$ is a transfer function, which means it is a complex value and frequency-dependent, we chose a frequency $w_{0}(100-200 \mathrm{~Hz})$ to minimize the off diagonal terms. The solution is shown in Eq. (4)

$$
\begin{array}{ll}
a=c=\frac{1}{(1+q)}, & b=d=1-a \\
e=f=\frac{1}{(1+p)}, & g=h=1-e
\end{array}
$$$$
p=\sqrt{\left|\frac{G_{11}\left(j w_{0}\right) G_{21}\left(j w_{0}\right)}{G_{22}\left(j w_{0}\right) G_{12}\left(j w_{0}\right)}\right|\left|\cos \left(\angle G_{11}\left(j w_{0}\right) G_{21}\left(j w_{0}\right)-\angle G_{22}\left(j w_{0}\right) G_{12}\left(j w_{0}\right)\right)\right|}
$$$$
q=\sqrt{\left|\frac{G_{11}\left(j w_{0}\right) G_{12}\left(j w_{0}\right)}{G_{22}\left(j w_{0}\right) G_{21}\left(j w_{0}\right)}\right|\left|\cos \left(\angle G_{11}\left(j w_{0}\right) G_{12}\left(j w_{0}\right)-\angle G_{22}\left(j w_{0}\right) G_{21}\left(j w_{0}\right)\right)\right|}
$$

We chose the frequency $w_{0}$ at the peak value of the offdiagonal terms $\left(G_{i j}, G_{j i}\right)$. After diagonalization, the magnitude of the off-diagonal terms $\left(\bar{G}_{i j}, \bar{G}_{j i}\right)$ near $w_{0}$ will be reduced. We diagonalize channels one, two, and three as channels $X, \theta$, and $\psi$, and we convert channels four and five to channels $Y$ and $\phi$. Channel 6 remains the same, but its notation is changed to channel $Z$. This process is performed on the actual system to obtain a diagonal system transfer function. It is important to note that the solution only minimizes the off-diagonal terms near $w_{0}$. We will then design the controller based upon the diagonalized system. The feedback signals are taken from the diagonalized system and the manipulated inputs from the controller are passed through a de-diagonalizing process to control the actual axis actuators.

\section{System identification}

In order to design a modern controller, we need a model of the system. A good model can reduce the uncertainty due to model mismatch. Although the DSMC controller will take care of any uncertainty, better identification will lead to a more robust controller. In the paper, we use curve fitting on the diagonalized system.

The six diagonal terms are now used for system identification. By using curve fitting, one can obtain very precise system transfer functions. Fig. 3 demonstrates the very good match between the modeled and the experimental step responses.

Due to space limitations, only the responses for Actuators 1 and 2 are shown. The system identification results are also shown in Fig. 4.

\section{Controller design}

\subsection{Discrete sliding mode control}

As described in Section 1, a discrete sliding mode controller has been constructed to achieve robust non-linear control performance and to meet the conditions for computer implementation. The design is based on equivalent control, including noise considerations [19]. The noise characteristics are determined from previous experiments.

Consider the discrete system as in Eq. (7),

$$
\begin{aligned}
& x(k+1)=(\Phi+\Delta \Phi) x(k)+\Gamma u(k)+\Gamma f(k) \\
& y(k)=(C+\Delta C) x(k)+v(k)
\end{aligned}
$$

where the state vector is $x(k) \in \mathfrak{R}^{n \times 1}$, the control effort is $u(k) \in \mathfrak{R}^{1}$, and the bounded disturbance is $f(k) \in \mathfrak{R}^{1}$.
$\Phi \in \mathfrak{R}^{n \times n}, \quad \Gamma \in \mathfrak{R}^{n \times 1}$, and $C \in \mathfrak{R}^{1 \times n}$ are the nominal or known system matrices; $\Delta \Phi \in \Re^{n \times n}$ and $\Delta C \in \Re^{1 \times n}$ are unmodeled uncertainties. Defining the sliding surface:

$s(k)=\Lambda x(k)$.

The DSMC will bring the state onto the manifold

$$
\begin{aligned}
& s(k+1)=\Lambda x(k+1) \\
& =\underbrace{\Lambda \Phi x(k)+\Lambda \Gamma u(k)}_{\text {known }}+\underbrace{\Lambda \Gamma f(k)+\Lambda \Delta \Phi x(k)}_{\text {unknown }}=0 .
\end{aligned}
$$

The equivalent control would be

$u_{\mathrm{eq}}(k)=-(\Lambda \Gamma)^{-1} \Lambda[\underbrace{\Phi x(k)}_{\text {known }}+\underbrace{\Gamma f(k)+\Delta \Phi x(k)}_{\text {unknown }}]$.

Since there is no a priori knowledge of $f(k)$, one can try substituting it with $f(k-1)$. In this way, one introduces a noise dynamic: $f(k)=f(k-1)$. The equivalent control effort becomes,

$$
\begin{aligned}
u_{\mathrm{eq}}(k)= & -(\Lambda \Gamma)^{-1} \Lambda[\Phi x(k)+\Gamma f(k-1)+\Delta \Phi x(k-1)] \\
u_{\mathrm{eq}}(k)= & -(\Lambda \Gamma)^{-1} \Lambda[\Phi x(k)+x(k)-\Phi x(k-1) \\
& -\Gamma u(k-1)] .
\end{aligned}
$$

Considering the effect of actuator saturation, the control effort can be designed as:

$u(k)= \begin{cases}u_{\mathrm{eq}}(k) & \left\|u_{\mathrm{eq}}(k)\right\| \leq u_{\max } \\ -u_{\max } \frac{u_{\mathrm{eq}}(k)}{\left\|u_{\mathrm{eq}}(k)\right\|} & \left\|u_{\mathrm{eq}}(k)\right\|>u_{\max } .\end{cases}$ 

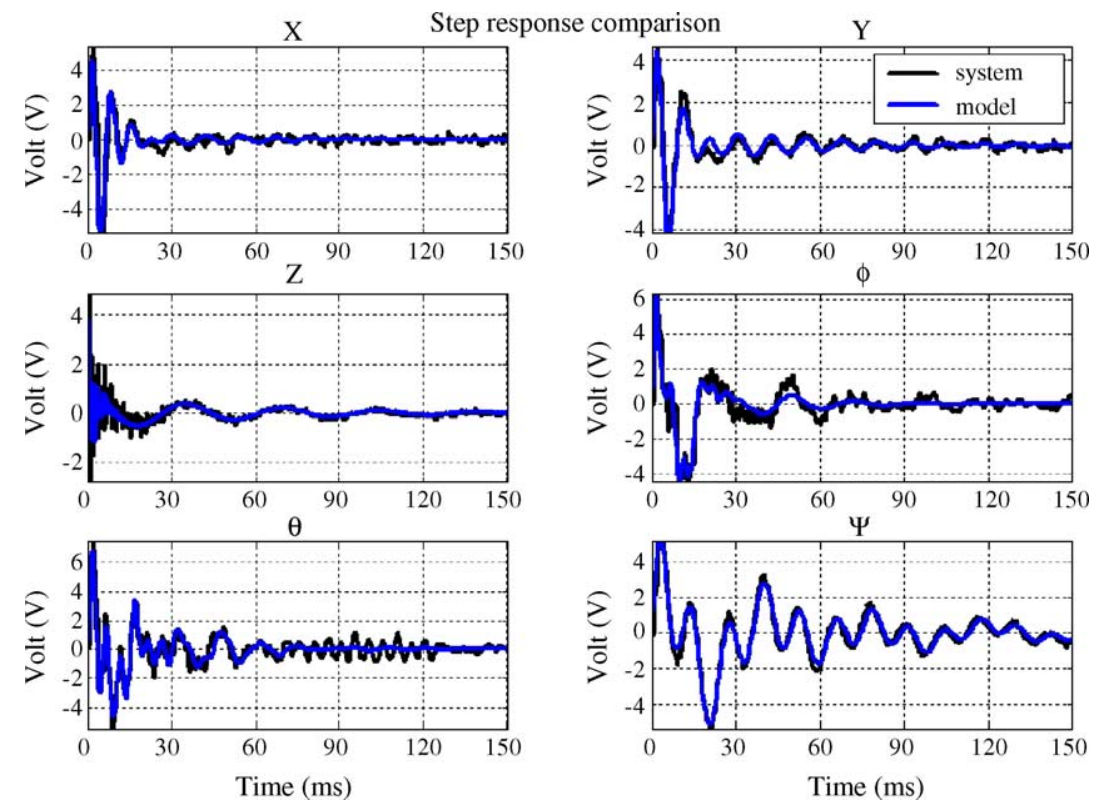

Fig. 3. Step response comparison. The dashed line is the experimental data and the solid line is the model response.

\subsection{Stability analysis}

Considering the region close to the sliding manifold, one has $\left\|u_{\mathrm{eq}}(k)\right\| \leq u_{\max }$. Substituting the control effort into Eq. (7), one gets $s(k+1)=\Lambda\{\Gamma[(f(k)-f(k-1))]+$ $\Delta \Phi[x(k)-x(k-1)]\}$; therefore, the state trajectory will converge to within a certain gap in this condition.

Now consider the condition where $\left\|u_{\mathrm{eq}}(k)\right\|>u_{\max }$. Thus, if the condition

$$
\begin{gathered}
u_{\max } \geq-(\Lambda \Gamma)^{-1} \Lambda[\Phi x(k)+x(k)-\Phi x(k-1) \\
-\Gamma u(k-1)], \quad \forall k \in[0, N]
\end{gathered}
$$
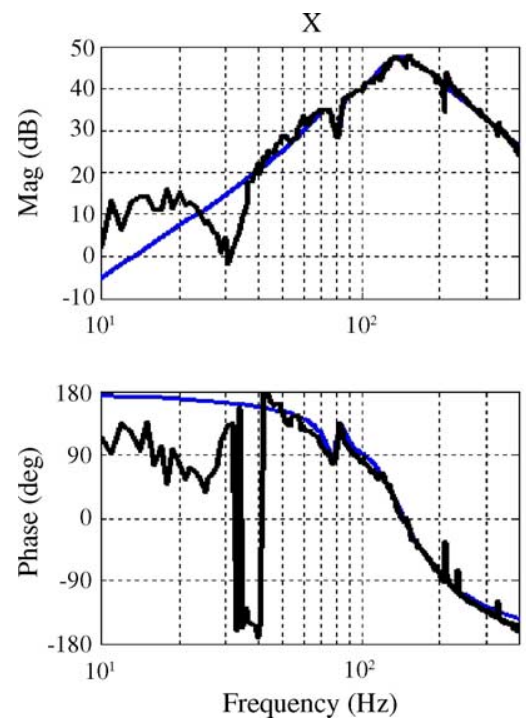

is true, the condition $\|s(k+1)\| \leq \| s(k)$ is also true. The value of $\|s(k)\|$ decreases monotonically and will converge to the unsaturated range with $\left\|u_{\mathrm{eq}}(k)\right\| \leq u_{\max }$ in a finite number of steps. The sliding function will again become $s(k+1)=\Lambda\{\Gamma[(f(k)-f(k-1))]+\Delta \Phi[x(k)-x(k-1)]\}$. If the disturbance is small, the state will stay close to the sliding manifold, within a gap. Notice that this controller does not use the switching function structure. There is no guarantee this controller can deal with parameter uncertainties and disturbances. The states would converge to a gap around the sliding manifold. The gap depends on parameter uncertainties and the disturbance.
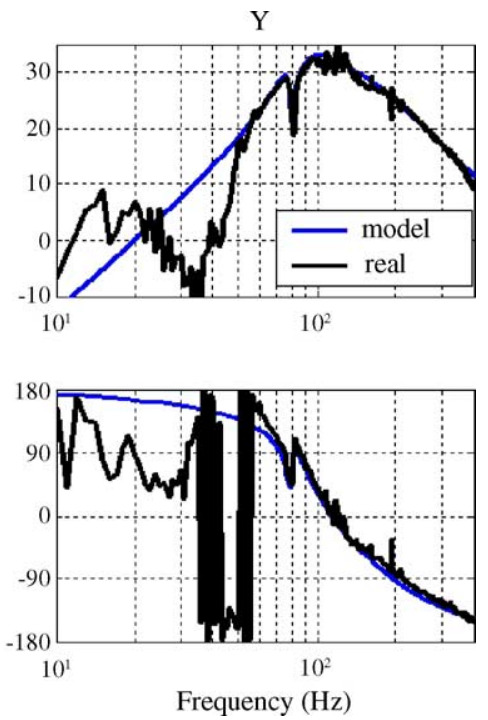

Fig. 4. System identification results for the diagonalized system of Actuators 1 and 2. 


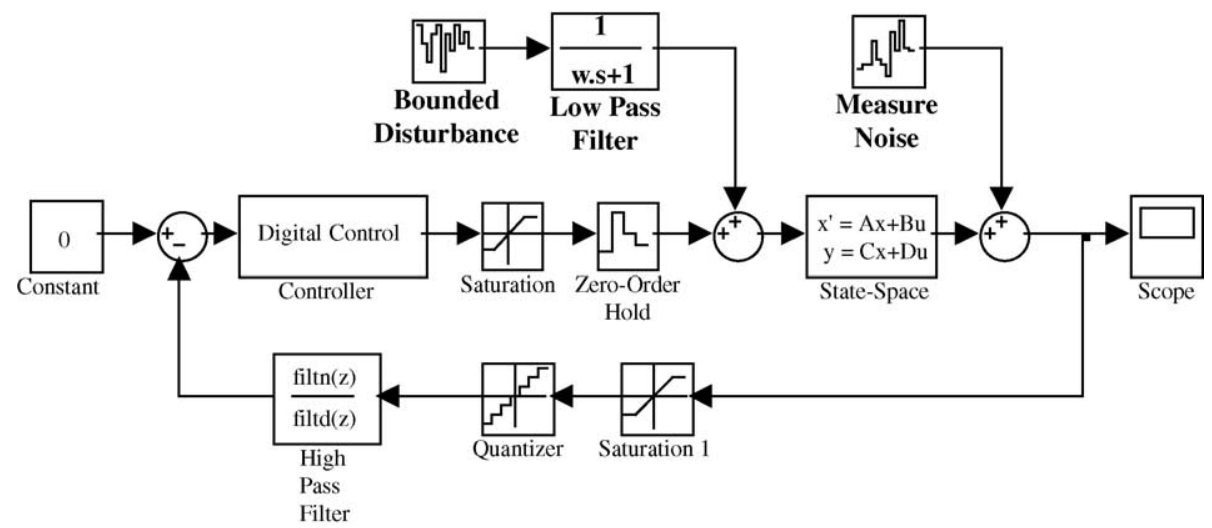

Fig. 5. The simulation block diagram.

In order to resolve $\Lambda$ the system can be described by Eq. (11):

$$
\begin{aligned}
z(k+1)=\left[\begin{array}{c}
z_{1}(k+1) \\
z_{2}(k+1)
\end{array}\right]= & {\left[\begin{array}{ll}
\Phi_{Z_{11}} & \Phi_{Z_{12}} \\
\Phi_{Z_{21}} & \Phi_{Z_{22}}
\end{array}\right]\left[\begin{array}{l}
z_{1}(k) \\
z_{2}(k)
\end{array}\right] } \\
& +\left[\begin{array}{l}
0 \\
I
\end{array}\right](u(k)+f(k)) \\
z(k+1)=\Phi_{Z} z(k+1)+ & \Gamma_{Z}(u(k)+f(k))
\end{aligned}
$$

where $z_{1}(k) \in \mathfrak{R}^{n-1}$ and $z_{2}(k) \in \mathfrak{R}^{1}$ are the system states, and $\Phi_{Z} \in \Re^{n \times n}, \quad \Phi_{Z_{11}} \in \Re^{(n-1) \times(n-1)}, \quad \Phi_{Z_{12} \in \Re^{(n-1) \times 1}}$, $\Phi_{Z_{21}} \in \Re^{1 \times(n-1)}, \Phi_{Z_{22} \in \Re^{1 \times 1}}$, and $\Gamma_{Z} \in \Re^{n \times 1}$ are system matrices. Defining the sliding surface:

$s(k)=K z_{1}(k)+z_{2}(k)=\Lambda z(k) \quad$ with $\Lambda=\left[\begin{array}{ll}K & 1\end{array}\right]$.

The DSMC will bring the state onto the manifold

$$
s(k)=0 \Rightarrow z_{2}(k)=-K z_{1}(k) \text {. }
$$

One can then use the optimal control technique to characterize the performance [20]

$J=\sum_{k=0}^{\infty} z_{1}(k)^{\mathrm{T}} Q z_{1}(k)+z_{2}(k)^{\mathrm{T}} R z_{2}(k)$

where $Q$ and $R$ are the weighting matrices. The variable $K$ can be determined by solving the Algebraic Riccati equation. The system on the manifold would behave as

$$
z_{1}(k+1)=\left(\Phi_{Z_{11}}-\Phi_{Z_{12}} K\right) z_{1}(k) .
$$

\section{Controller simulation and implementation}

\subsection{Simulation}

The simulations and experiments compare the results from a discrete lead-lag controller and the discrete sliding mode
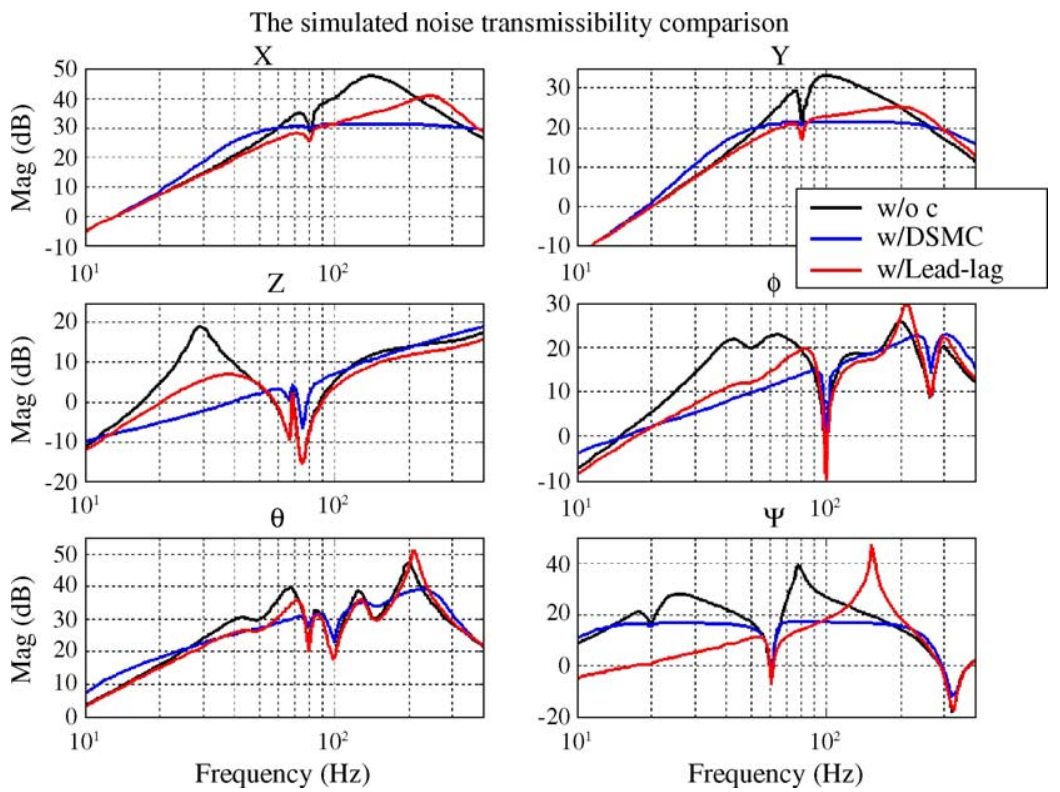

Fig. 6. The simulated noise transmissibility comparison between the DSMC controller and a lead-lag compensator. 

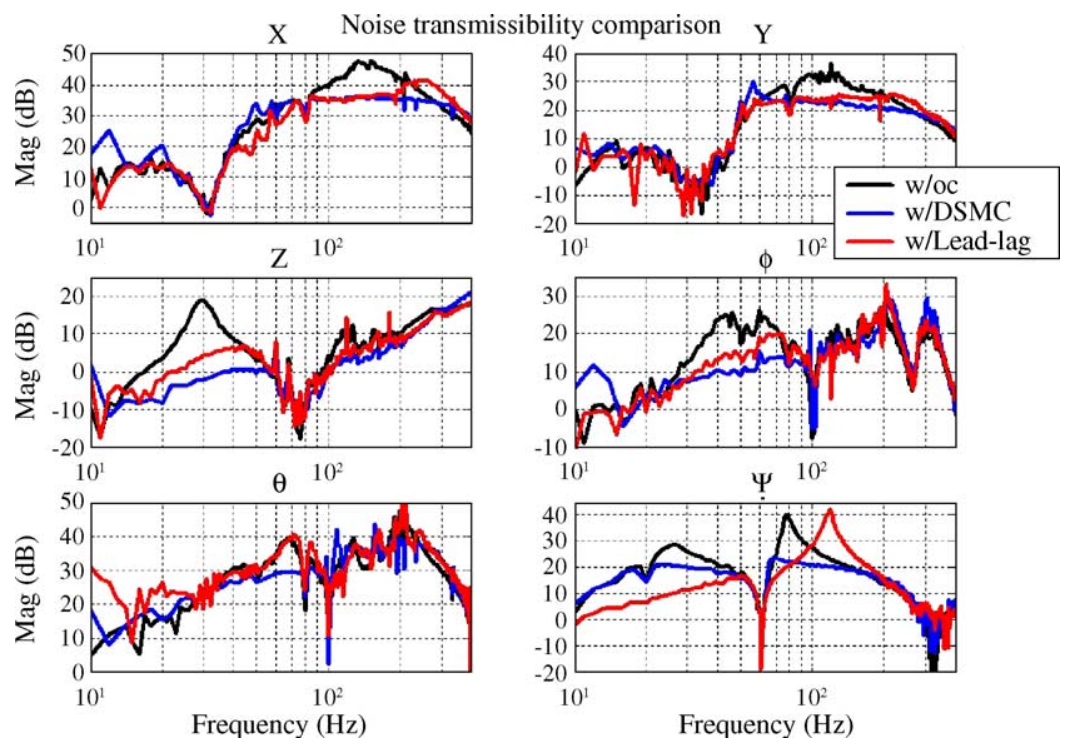

Fig. 7. Noise transmissibility with DSMC and lead-lag controller. The solid line is taken with DSMC controller; the dotted line is taken without any controller; the dashed line is taken with the lead-lag compensator.

controller. The simulation block structure is shown in Fig. 5 . The simulation includes the effect of the Zero Order Hold $(\mathrm{ZOH})$, the quantizer, bounded disturbances, and the measurement noise. The accelerometer signal goes through a high pass filter to remove the dc offset.

Fig. 6 shows the comparison between the noise transmissibility with the DSM controller and a lead-lag compensator, after tuning to the best parameters. The result shows that DSMC decreases the noise in the control band without increasing the noise in the high frequency range. The lead-lag compensator is able to attenuate the noise level in most of the operating frequency band, but it also increases the higher frequency noise.

\subsection{Experiment}

The experiment uses a slight modification to the DSMC implementation. Because the servo bandwidth is very high (up to $4.4 \mathrm{kHz}$ ) and there is a resonance around $400 \mathrm{~Hz}$, the control uses a low-pass filter on the $Z, \varphi$, and $\theta$ systems. The DSMC design procedure is then carried out on the filtered systems. A noise transmissibility comparison between the DSMC and the lead-lag compensator is shown in Fig. 7. With DSMC control, there is attenuation of $20 \mathrm{~dB}$ in channel $Z$ at $30 \mathrm{~Hz}$ and of about $10 \mathrm{~dB}$ in the other channels.

The noise transmissibility with the lead-lag compensator shows that noise is decreased at $130 \mathrm{~Hz}$ for $X$ and $Y$ motion.
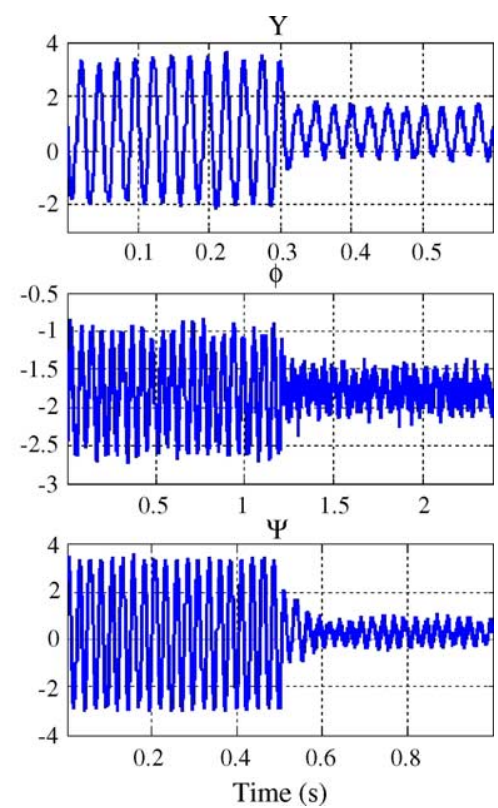

Fig. 8. Acceleration signals under a sinusoidal excitation. 

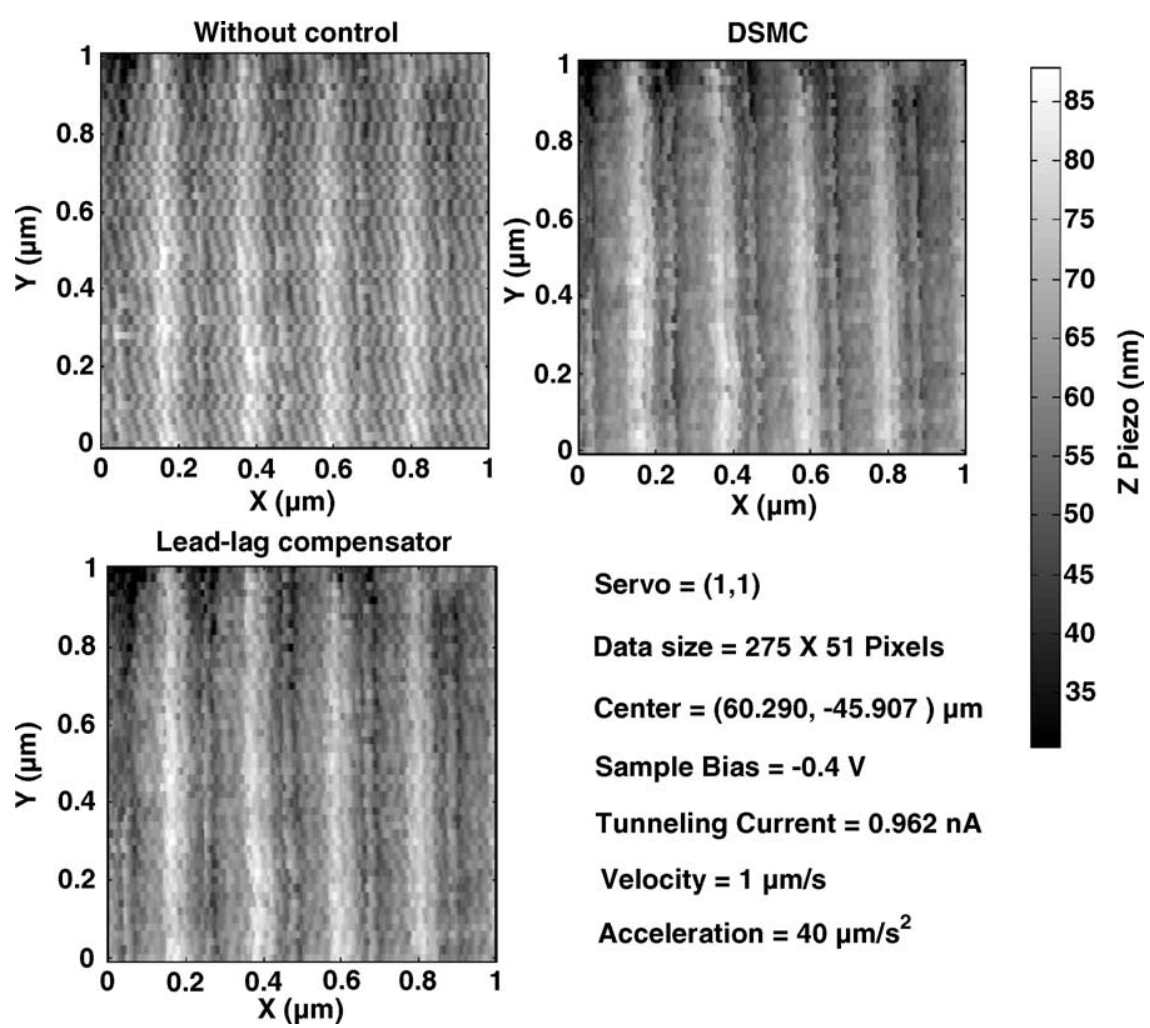

Fig. 9. $\mathrm{M}^{3}$ image comparison with a fixed sine disturbance, comparing no active vibration control, DSMC, and lead-lag compensator.

The controller also decreases noise at $30 \mathrm{~Hz}$ for $Z$ motion. However, it increases the noise in channels $X, Y, \varphi, \theta$, and $\Psi$ at $230 \mathrm{~Hz}, 230 \mathrm{~Hz}, 200 \mathrm{~Hz}, 200 \mathrm{~Hz}$, and $110 \mathrm{~Hz}$, respectively.

By injecting a sinusoidal signal into the system (Fig. 8), one can also observe that the control is effective in suppressing vibrations.

The disturbance frequencies applied to channels $X, Y, Z, \varphi$, $\theta$, and $\Psi$ are $138 \mathrm{~Hz}, 128 \mathrm{~Hz}, 30 \mathrm{~Hz}, 46 \mathrm{~Hz}, 68 \mathrm{~Hz}$, and $78 \mathrm{~Hz}$, respectively. Acceleration in $z$ is reduced from $7.5 \mathrm{~mm} / \mathrm{s}^{2}$ to $1 \mathrm{~mm} / \mathrm{s}^{2}$.

Overall, the DSMC is better than the lead-lag controller; it has better attenuation and produces less additional noise. Fig. 9 compares the SPM images taken with and without using the lead-lag compensator and the DSMC for vibration control. The sample was made by Laser Focused Atom Deposition (LFAD) [21]; the pitch is $212 \mathrm{~nm}$. The images were taken using a high scan velocity $(1 \mu \mathrm{m} / \mathrm{s})$. Because the STM is already under the protection of very sophisticated passive isolation, some extra disturbance at $30 \mathrm{~Hz}$ was added to show the effectiveness of the active suspension. The images demonstrate the performance of the DSMC controller. It results in a clearer, smoother image.

\section{Conclusion}

An active vibration isolation system was installed on the $\mathrm{M}^{3}$ system at NIST using a discrete sliding mode controller.
The DSMC design is based on frequency domain system identification. Because the active isolation system has six piezoelectric actuator inputs and six accelerometer outputs, it is necessary to reduce the system complexity for practical implementation. This paper introduces a first-level decomposition based on the geometric system constraints. The linear system decoupling technique is then applied to diagonalize the system transfer function matrix. Experimental verification is carried out to examine the validity of the reduction. The DSMC implementation is experimentally compared with a conventional, digital, lead-lag compensator. The control results and the SPM imaging performance both demonstrate the effectiveness of the proposed DSMC implementation.

\section{Acknowledgements}

Technical assistance from Bill Penzes, George Witzgall, and Vince Scheuerman are gratefully acknowledged.

\section{References}

[1] G. Binning, H. Rohrer, Scanning tunneling microscopy, Helvetica Phys. Acta 55 (1982) 726-735.

[2] D. Kobayashi, H. Fujita, A micromachined unit for tunnel current control, in: Proceedings of the 1995 INRIA/IEEE Symposium on Emerging Technologies and Factory Automation, vol. 2, Paris, France, October 10-13, 1995, pp. 277-285. 
[3] R.P. Ried, H.J. Mamin, B.D. Terris, L.S. Fan, D. Rugar, $5 \mathrm{MHz}$, $2 \mathrm{~N} / \mathrm{m}$ piezoresistive cantilevers with INCISIVE tips, in: Proceedings of the International Conference on Solid State Sensors Actuators, Chicago, IL, USA, June 16-19, 1997, pp. 447-450.

[4] R.M.D. Stevens, N.A. Frederick, B.L. Smith, D.E. Morse, G.D. Stucky, P.K. Hansma, Carbon nanotubes as probes for atomic force microscopy, Nanotechnology 11 (2000) 1-5.

[5] J.A. Turner, J.S. Wiehn, Sensitivity of flexural and torsional vibration modes of atomic force microscope cantilevers to surface stiffness variations, Nanotechnology 12 (2001) 322-330.

[6] J.B. Thompson, B. Drake, J.H. Kindt, J. Hoskins, P.K. Hansma, Assessing the quality of scanning probe microscope designs, Nanotechnology 12 (2001) 394-397.

[7] Y.S. Kim, H.J. Nam, S.M. Cho, D.C. Kim, J.U. Bu, A self-actuating PZT cantilever integrated with piezoresistor sensor for AFM with high speed parallel operation, in: Proceedings of the IEEE conference on Micro Electro Mechanical Systems (MEMS), Las Vegas, NV, USA, January 20-24, 2002, pp. 689-692.

[8] W.J. Chang, Sensitivity of vibration modes of atomic force microscope cantilevers in continuous surface contact, Nanotechnology 13 (2002) 510-514.

[9] P.K. Cheng, K. Yackoboski, G.C. McGonigal, D.J. Thomson, A digital signal processor based atomic force microscope controller, in: Proceedings of the IEEE WESCANEX Communications, Power and Computing Conference. Part 2 (of 2), Winnipeg, Manitoba, Canada, May 15-16, 1995, pp. 456-461.

[10] H. Jung, J.Y. Shim, D. Gweon, Tracking control of piezoelectric actuators, Nanotechnology 12 (2001) 14-20.

[11] D. Gilsinn, M. Lyons, F. Scire, E.C. Teague, E. Amatucci, T. McIntyre, Design and Assembly of a Vibration Isolation System for the Molecular Measuring Machine, Draft National Institute of Standards and Technology Interagency Report, private communication, August 1991.

[12] J.A. Kramar, E. Amatucci, D. Gilsinn, J. Jun, W. Penzes, F. Scire, E.C. Teague, J. Villarrubia, Toward nanometer accuracy measurements, in: Proceedings of the SPIE International Society on Optical Engineering, vol. 3677, Santa Clara, CA, USA, March 15-18, 1999, pp. 1017-1028.

[13] S.Z. Sarpturk, Y. Istefanopulos, O. Kaynak, On the stability of discrete-time sliding mode control systems, IEEE Trans. Autom. Control 32 (1987) 930-932.

[14] G. Bartolini, A. Ferrara, V.I. Utkin, Adaptive sliding mode control in discrete-time systems, Automatica 31 (1995) 769-773.

[15] W. Gao, Y. Wang, A. Homaifa, Discrete-time variable structure control systems, IEEE Trans. Ind. Electron. 42 (1995) 117122.

[16] R.S. Whipple, The design and construction of scientific instruments, Trans. Opt. Soc. 22 (1920) 48-49.

[17] K.J. Lan, J.Y. Yen, J.A. Kramar, Sliding mode control for active vibration isolation of a long range scanning tunneling microscope, Rev. Sci. Instrum. 75 (2004) 4367-4373.
[18] K.J. Lan, J.Y. Yen, J.A. Kramar, Active vibration isolation for a long range scanning tunneling microscope, Asian J. Control 6 (2004) 179-186.

[19] K.D. Young, V.I. Utkin, Ü. Özgüner, A control engineer's guide to sliding mode control, IEEE Trans. Control Syst. Technol. 7 (1999) 328-342.

[20] F.L. Lewis, V.L. Syrmos, Optimal Control, second ed., Wiley-Interscience, New York, 1995, pp. 161-196.

[21] J.J. McClelland, R.E. Scholten, E.C. Palm, R.J. Celotta, Laser focused atomic deposition, Science 262 (1993) 877-880.

\section{Biographies}

Jia-Yush Yen became a member of IEEE in 1987. He was born in Taipei, Taiwan. He received the BS degree in mechanical engineering from $\mathrm{Na}-$ tional Tsinghua University, Hsinchu, Taiwan, in 1980, and the MS degree from University of Minnesota, MN, in 1983. In 1989, he received the PhD degree in mechanical engineering from University of California, Berkeley, CA. During his study at Berkeley, he received the IBM Graduate Fellowship in 1984-1985. Since 1989, he has been with National Taiwan University, Taipei, Taiwan, where he is currently a professor of mechanical engineering. He served as the treasurer of the Control System Chapter in IEEE Taipei Section in 1992. He also served as consultant for many companies including Industrial Technology Research Institute, Taiwan, and C Sun MFG Ltd., Taiwan. His research interests are in the areas of modeling and control of electromechanical systems, especially in precision control of computer peripherals and precision measurement systems. $\mathrm{He}$ is also a member of the ASME.

Kuo-Jung Lan received his $\mathrm{BS}$ degree and his $\mathrm{PhD}$ degree from $\mathrm{Na}-$ tional Taiwan University in 2000 and 2004, respectively. He then join the MediaTek Inc. as a senior research engineer. His research interests include automatic control, active vibration isolation technique and scanning probe microscopy. His theoretical research interests include PID control, LQG control, continuous/discrete sliding mode control, mu-synthesis, and robust control.

John A. Kramar received a BS in chemistry in 1983 from Abilene Christian University and a PhD in chemistry in 1990 from the California Institute of Technology. His thesis subject was scanning tunneling microscopy and spectroscopy of molybdenum disulfide. In 1990, he joined the National Institute of Standards and Technology to work on the Molecular Measuring Machine Project, of which he is now project leader. His current research interests are in nanometrology, particularly of length and force, and in molecular nanotechnology. Specific applications are scanned probe microscope-based nanolithography for the development of calibration standards, and the examination of prospective intrinsic atom-based dimensional standards. Dr. Kramar has been the recipient of U.S. Department of Commerce Gold and Silver Medal Awards for distinguished achievement in research. 